\title{
A comparative study on the effect of music therapy alone and a combination of music and yoga therapies on the psycho- physiological parameters of cardiac patients posted for angiography
}

\author{
Ajmera $\mathbf{S}^{1, *}$, Sundar $\mathbf{S}^{1}$, Amirtha Ganesh $\mathbf{B}^{2}$, Bhavanani A. $\mathbf{B}^{3}$, Dayanidy $\mathbf{G}^{3}$, \\ Ezhumalai $\mathbf{G}^{4}$
}

${ }^{1}$ Center for Music Therapy

Education and Research

(CMTER),

${ }^{2}$ Department of Cardiology,

Mahatma Gandhi Medical

College and Research Institute,

${ }^{3}$ Center for Yoga Therapy

Education and Research

(CYTER),

${ }^{4}$ Office of the Deanery of

Research,

Sri Balaji Vidyapeeth,

(Deemed to be University),

Pillaiyarkuppam,

Puducherry-607402.

For Correspondence

${ }^{*}$ Dr. Sundar S,

Email:sumusundhar@yahoo.com

Date of

Submisssion: 29-08-2018

Acceptance: 03-10-2018

\section{Access this article online}

Quick Response Code

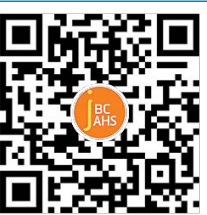

https://www.jbcahs.org

E-ISSN: 2581-6039

\section{ABSTRACT}

Background and Objectives: Patients undergoing cardiac catheterization and coronary angiography often experience high levels of anxiety and physiological disturbances. Music therapy and music interventions have been found to be effective in bringing down the anxiety and reducing the physiological disturbances for these patients. However, the efficacy of combination of music and yoga therapies for preprocedural anxiety and physiological disturbances needs to be studied. We aimed in this study to compare the effect of music therapy with the combination of music and yoga therapies to impact the psycho-physiological responses like anxiety, blood pressure, pulse rate and respiratory rate of patients who were posted for coronary angiography

Material and Methods: A total of 45 patients who were posted for coronary angiography were included in the study and randomly divided into three groups. Music therapy group $(n=16)$ received music listening intervention in the form listening to pre-recorded, patient-preferred, relaxing raga improvisational music for 15 minutes 1) on the previous day of angiography and 2) 15 minutes before being taken to the catheterization lab on the day of the angiography. The combination of music and the yoga group received both music therapy and yoga therapy in the form of pranava pranayama together for 15 minutes. The control group received only the standard medical treatment. The state of anxiety was measured by a five point single item Likert scale and the physiological measures such as systolic blood pressure (SBP), diastolic blood pressure (DBP), pulse rate (PR) and respiratory rate $(R R)$ were also recorded for the study

Results: Both music therapy alone and the combination of music and yoga therapies resulted in within the group significant reduction in anxiety and respiratory rate and music therapy group recorded additionally significant reduction in SBP, DBP and PR scores during the period of intervention.

Conclusion: Our findings indicate that music therapy alone can bring down the anxiety levels and reduce the physiological disturbances of patients posted for angiography. Also, combination of music and yoga therapies can bring down the anxiety levels and improve the deep breathing pattern for these patients posted for angiography. More studies are needed to confirm these findings.

\section{Keywords}

Music, Yoga, Cardiac Catheterization, Angiogram, Anxiety

\section{INTRODUCTION}

Patients having acute coronary syndrome (ACS) and waiting for the cardiac catheterization and coronary angiography (CAG) procedures may experience high levels of anxiety and stress. Long waiting time, nonavailability of psycho-social support, first time schedule to angiography and uncertainty about the procedural outcome are some of the reasons why patients in the pre-catheterization period experience fear, anxiety and stress. Unrelieved anxiety and stress during this period may not only lead to lack of co-operation by the patients. Patients also suffer physiological disturbances, reduced immune response
How to Cite: Ajmera S, Sundar S, Amirtha Ganesh B, Bhavanani AB, Dayanidy G, Ezhumalai G. A comparative study on the effect of music therapy alone and a combination of music and yoga therapies on the psycho-physiological parameters of cardiac patients posted for angiography. J Basic Clin Appl Health Sci. 2018;1:13-8 
and altered cardiovascular functioning like tachycardia, increase in core body temperature, heightened blood pressure and nausea which may directly impact the clinical outcomes. ${ }^{1-3}$ Hence, it becomes important to adopt a multidisciplinary strategy to minimize anxiety and physiological disturbances of in-patients posted for CAG. Use of complementary therapies like music and yoga as stand- alone adjunct therapies have been studied to be beneficial in reducing stress and anxiety by encouraging the patients to relax, slow down the breathing and activate the para-sympathetic system and inhibiting the sympathetic nervous system. ${ }^{4-6}$ However, we did not come across any study reported so far on evaluating the effects of the combination of yoga and music therapies as procedural support in $C A G$ patients.

A Cochraine systematic review which included 26 studies with the total of 2051 participants published in the year 2013 concluded that music listening interventions in which pre-recorded music was used by health care and allied health care professionals reduced significantly pre-operative anxiety in medical patients and hence music could serve as a good alternative option to sedatives and anti-anxiolytic drugs. ${ }^{5}$

Pranava Pranayama is slow, deep and rhythmic breathing where emphasis is placed on making the sound AAA, UUU and MMM while breathing out for duration of 2 to 3 times the duration of the inhaled breath. ${ }^{6,7}$ It is a four part technique consisting of lower chest breathing with the sound of AAA, midchest breathing with the sound of UUU, upper chest breathing with the sound of MMM and then the union of the earlier three parts in a complete yogic breath with the sound of AAA, UUU and MMM.

Previous studies by Bhavanani and colleagues have reported that pranayama-based breathing and relaxation is effective in reducing blood pressure and stress $^{6-8}$. It was studied in hypertensive and diabetic patients and found to reduce heart rate and SBP within five minutes of the practice as well as reduce stress and anxiety over 12 weeks of training. This was attributed to normalization of autonomic cardiovascular rhythms as a result of increased vagal modulation and/or decreased sympathetic activity and improved baroreflex sensitivity.

It has been reported that the effects of pranava relaxation in supine position are strikingly similar to the deep relaxation technique (DRT) popularized by Swami Vivekananda Yoga Anusandhana Samsthana (SVYASA)
University, Bangalore. ${ }^{9}$ Studies from SVYASA have reported that DRT improves sustained attention and reduces the state of anxiety while reducing cardiac sympathetic activity. ${ }^{9,10}$

We conducted this trial to compare the effect of music therapy with that of the combination of music and yoga therapies on the psycho-physiological parameters like anxiety, systolic blood pressure, diastolic blood pressure, pulse rate and respiratory rate in patients posted for coronary angiography as no studies are reported on this combination intervention strategy. We used pre-recorded, patient-preferred, instrumental, Indian classical music as a music listening intervention. Though music therapists provide mostly customized live musical experiences in therapy sessions based on the context in which they are administering the therapy, it is not totally uncommon for qualified music therapists working in hospital settings to use music listening interventions using pre-recorded music in their research studies. ${ }^{11,12}$ For yoga therapy, we used pranava pranayama breathing technique.

\section{MATERIAL AND METHODS}

The study was approved by the Institutional Human Ethics Committee of Mahatma Gandhi Medical College and Research Institute, Pondicherry. This open label, three-arm, randomized controlled study was carried out for a period of 4 months in the catheterization laboratory attached to the Department of Cardiology of the hospital by a qualified music therapist. All patients in the age group of 30 to 65 who were posted for angiography for the first time and gave written informed consent, understood the local language and be able to take instructions were enrolled for the study. Patients who received any psychiatric treatment or anxiolytic medication, hard of hearing, having a diagnosis of cerebrovascular accident (CVA) or scheduled for an emergency angiogram were excluded from the study.

For sample size calculation, we assumed a type 1 error of 0.05 ( $95 \%$ confidence interval) by keeping $\alpha$ $=0.01$ and type II error of $\beta=0.02$ (a power of $80 \%$ ). The sample size computed was 30 in each group with a total of 90 .

In total, 45 patients who fulfilled the inclusion criteria were randomly assigned by a computer assisted method in which random numbers were generated in the system and accordingly the patients were distributed into the three groups with 16 in the 
music therapy group, 14 in the combination of music and yoga therapy group and 15 in the control group. The random allocation sequence was concealed in sealed opaque covers and kept in the cardiothoracic and vascular surgery (CTVS) ward. The baseline characteristics indicated that the three groups were identical and hence could be compared. The patients participating in the study were informed about the study procedure and were free to withdraw from the study at any point of time they wanted. There were two interventions for both the groups. The first was on the previous day to angiography and the second was on the day of the angiography. The first intervention was administered in the CTVS ward and the second intervention was administered in the waiting area of the catheterization laboratory.

Participants in the music therapy group listened to preferred, instrumental, Indian classical, relaxing prerecorded improvisational raga music for 15 minutes on the day 1 and also on the day of the angiography for 15 minutes in the presence of a qualified music therapist. In the combination of music and yoga therapy group, the patients were engaged in yogic breathing for 7.5 minutes by a qualified yoga therapist followed by 7.5 minutes of music listening intervention as was in the music group. For both the interventions, the base line and the end of study measures were recorded. Anxiety was measured by a single item five point Likert scale having the options from "no anxiety, mild anxiety, moderate anxiety, severe anxiety and extremely high anxiety." The Likert scale was used as it is useful to record the anxiety levels easily and quickly and also when the researchers have very short time or psychometric tool space and need to reduce the burden on the patients waiting for an invasive interventional procedure evoking high levels of anxiety. ${ }^{13}$ Blood pressure and pulse rate were recorded by electronic sphygmomanometer.

Distribution of data for the study was checked by performing normality tests and data was found to be normally distributed. Hence data was analysed by paired t tests, ANOVA and repeated measures ANOVA. Wilcoxon test and Friedman's test was conducted for anxiety.

Table 1: Comparison of pre and post measures of selected psycho-physiological parameters in all the three groups control $(n=15)$, music (16) and music and yoga groups $(n=14)$

\begin{tabular}{|c|c|c|c|c|c|c|c|c|c|}
\hline \multirow{3}{*}{ Measurement } & \multicolumn{9}{|c|}{ Groups } \\
\hline & \multicolumn{3}{|c|}{ Control $(n=15)$} & \multicolumn{3}{|c|}{ Music $(n=16)$} & \multicolumn{3}{|c|}{ Music and Yoga $(n=14)$} \\
\hline & $\begin{array}{c}\text { Pre } \\
\text { Mean } \pm \text { SD }\end{array}$ & $\begin{array}{c}\text { Post } \\
\text { Mean } \pm \text { SD }\end{array}$ & $p$-value & $\begin{array}{c}\text { Pre } \\
\text { Mean } \pm \text { SD }\end{array}$ & $\begin{array}{c}\text { Post } \\
\text { Mean } \pm \text { SD }\end{array}$ & $p$-value & $\begin{array}{c}\text { Pre } \\
\text { Mean } \pm \text { SD }\end{array}$ & $\begin{array}{c}\text { Post } \\
\text { Mean } \pm \text { SD }\end{array}$ & $p$-value \\
\hline \multicolumn{10}{|l|}{ Day 1} \\
\hline SBP & $117.53 \pm 12.61$ & $114.40 \pm 10.29$ & 0.32 & $133.31 \pm 18.56$ & $127.00 \pm 19.14$ & 0.32 & $131.07 \pm 27.1$ & $127.14 \pm 20.3$ & 0.29 \\
\hline DBP & $71.87 \pm 12.01$ & $70.27 \pm 9.12$ & 0.57 & $80.38 \pm 6.14$ & $76.81 \pm 8.53$ & 0.04 & $80.43 \pm 14.16$ & $77.93 \pm 12.23$ & 0.23 \\
\hline PR & $67.80 \pm 10.57$ & $69.13 \pm 88.14$ & 0.45 & $78.12 \pm 12.01$ & $67.44 \pm 13.32$ & 0.04 & $73.93 \pm 11.45$ & $68.43 \pm 19.29$ & 0.12 \\
\hline $\mathrm{RR}$ & $22.67 \pm 4.19$ & $21.40 \pm 4.70$ & 0.13 & $23.93 \pm 4.26$ & $22.07 \pm 6.02$ & 0.04 & $23.93 \pm 4.27$ & $22.07 \pm 4.75$ & 0.02 \\
\hline Anxiety & $0.60 \pm 0.83$ & $0.67 \pm 0.90$ & 0.32 & $0.88 \pm 0.81$ & $0.06 \pm 0.25$ & 0.001 & $0.93 \pm 0.99$ & $0.43 \pm 0.75$ & 0.04 \\
\hline \multicolumn{10}{|l|}{ Day 2} \\
\hline SBP & $132.71 \pm 29.38$ & $130.79 \pm 24.37$ & 0.22 & $131.62 \pm 16.34$ & $126.25 \pm 15.64$ & 0.01 & $132.71 \pm 29.38$ & $130.79 \pm 24.37$ & 0.42 \\
\hline DBP & $76.73 \pm 12.38$ & $73.73 \pm 11.32$ & 0.09 & $80.19 \pm 4.92$ & $76.81 \pm 5.14$ & 0.001 & $79.57 \pm 11.04$ & $78.64 \pm 13.48$ & 0.68 \\
\hline PR & $73.67 \pm 11.81$ & $69.53 \pm 9.98$ & 0.12 & $76.12 \pm 11.93$ & $71.31 \pm 10.72$ & 0.01 & $76.12 \pm 14.70$ & $71.31 \pm 13.87$ & 0.07 \\
\hline $\mathrm{RR}$ & $22.67 \pm 4.189$ & $20.80 \pm 3.30$ & 0.12 & $23.75 \pm 4.34$ & $19.69 \pm 3.14$ & 0.01 & $23.93 \pm 4.27$ & $20.00 \pm 4.08$ & 0.001 \\
\hline Anxiety & $0.53 \pm 0.74$ & $0.53 \pm 0.74$ & 1.00 & $0.67 \pm 0.70$ & $0.25 \pm 0.44$ & 0.01 & $0.64 \pm 0.84$ & $0.29 \pm 0.61$ & 0.03 \\
\hline
\end{tabular}


Table 2: Comparison of SBP, DBP, PR, RR and anxiety levels from baseline to day 2 post for each group.

\begin{tabular}{|c|c|c|c|c|c|c|c|}
\hline \multirow{3}{*}{ Parameter } & \multirow{3}{*}{$\begin{array}{c}\text { Days of } \\
\text { measurement }\end{array}$} & \multicolumn{6}{|c|}{ Groups } \\
\hline & & \multicolumn{2}{|c|}{ Control } & \multicolumn{2}{|c|}{ Music } & \multicolumn{2}{|c|}{ Music and yoga } \\
\hline & & Mean \pm SD & $p$-value & Mean \pm SD & $p$-value & Mean \pm SD & $p$-value \\
\hline \multirow{3}{*}{ SBP } & pre Day1 & $117.53 \pm 12.60$ & & $133.31 \pm 18.56$ & & $131.07 \pm 27.12$ & \\
\hline & post Day1 & $114.40 \pm 10.29$ & 0.367 & $127.00 \pm 19.14$ & 0.15 & $127.14 \pm 20.33$ & 0.42 \\
\hline & post Day2 & $118.40 \pm 11.54$ & & $126.25 \pm 15.64$ & & 130.7924 .37 & \\
\hline \multirow{3}{*}{ DBP } & Pre Day 1 & $71.87 \pm 12.01$ & & $80.38 \pm 6.14$ & & $80.43 \pm 14.16$ & \\
\hline & Post Day1 & $70.27 \pm 9.12$ & 0.382 & $76.81 \pm 8.52$ & 0.07 & $77.93 \pm 12.23$ & 0.39 \\
\hline & Post Day2 & $73.73 \pm 11.32$ & & $76.81 \pm 5.14$ & & $78.64 \pm 13.48$ & \\
\hline \multirow{3}{*}{ PR } & Pre Day1 & $67.80 \pm 10.51$ & & $78.12 \pm 12.01$ & & $73.93 \pm 11.45$ & \\
\hline & Post Day 1 & $69.13 \pm 8.85$ & 0.775 & $67.44 \pm 13.32$ & 0.001 & $68.43 \pm 10.28$ & 0.14 \\
\hline & Post Day2 & $69.53 \pm 9.97$ & & $69.53 \pm 9.97$ & & $69.57 \pm 13.86$ & \\
\hline \multirow{3}{*}{$\mathrm{RR}$} & Pre Day1 & $22.67 \pm 4.19$ & & $23.75 \pm 4.34$ & & $23.93 \pm 4.26$ & \\
\hline & Post Day1 & $21.40 \pm 4.70$ & 0.242 & $23.00 \pm 6.02$ & 0.01 & $22.07 \pm 4.74$ & 0.001 \\
\hline & Post Day2 & $20.80 \pm 3.30$ & & $19.69 \pm 3.14$ & & $20.00 \pm 4.08$ & \\
\hline \multirow{3}{*}{ Anxiety } & Pre Day1 & $0.60 \pm 0.83$ & & $0.88 \pm 0.80$ & & $0.94 \pm 0.00$ & \\
\hline & Post Day 1 & $0.67 \pm 0.90$ & 0.001 & $0.06 \pm 0.25$ & 0.001 & $0.43 \pm 0.76$ & 0.001 \\
\hline & Post Day2 & $0.53 \pm 0.74$ & & $0.25 \pm 0.45$ & & $0.29 \pm 0.61$ & \\
\hline
\end{tabular}

\section{RESULTS}

The pre and post measurement of SBP, DBP, PR, $\mathrm{RR}$ and anxiety among patients who had undergone coronary angiography were measured and tested for any possible difference. On day1, the level of SBP, DBP, $\mathrm{PR}$ and $\mathrm{RR}$ remained unaltered in the control group which received only routine treatment. In the music therapy group, the DBP, PR and RR of the patients had significantly reduced from 80 to 77,78 to 67,24 to 22 and 0.88 to 0.06 respectively ( $\mathrm{p}=0,04, \mathrm{p}=0.04$, $\mathrm{p}=0.04, \mathrm{p}=0.001)$. In the combination of music and yoga therapies group, the RR had marginally reduced from 24 to 22 and it was significant $(\mathrm{p}=0.02)$. In this group, anxiety was also significantly reduced from 0.93 to $0.43(\mathrm{p}=0.04)$. On day2, the level of SBP, $\mathrm{DBP}, \mathrm{PR}, \mathrm{RR}$ and anxiety remained unaltered in the control group. In music therapy group, the SBP, DBP, PR, RR and anxiety levels of the patients had significantly reduced from 132 to 126,80 to 77,76 to 71,23 to 19 and 0.67 to 0.25 respectively $(\mathrm{p}=0.01$, $\mathrm{p}=0.001, \mathrm{p}=0.01, \mathrm{p}=0.01, \mathrm{p}=0.01)$. In the combination of music and yoga therapy group, RR had marginally reduced from 24 to 20 and anxiety was reduced from 0.64 to 0.29 and this differences were significant $(\mathrm{p}=0.001, \mathrm{p}=0.03)$. (Table 1$)$

The mean levels of SBP, DBP, PR, RR and anxiety at day 1 pre, day 1 post and day 2 post for each group was analysed to find the variations over these periods. In control group, the mean reduction of SBP, DBP, $\mathrm{PR}, \mathrm{RR}$ were not significantly change in two days, but anxiety alone was reduced from 0.6 to 0.53 and significant $(\mathrm{p}=0.001)$. In music group, SBP \& DBP were not significantly changed but PR, RR and anxiety were reduced from 78 to 69,24 to 20 and 0.88 to 0.25 respectively. $(\mathrm{p}=0.001, \mathrm{p}=0.01, \mathrm{p}=0.001)$. In music 
Table 3: Post-hoc test for music and music with yoga group

\begin{tabular}{|c|c|c|c|c|c|c|}
\hline \multirow{2}{*}{ Group } & \multirow{2}{*}{ Parameter } & \multirow{2}{*}{ Between pairs } & \multirow{2}{*}{ Mean diff } & \multicolumn{2}{|c|}{$95 \% \mathrm{Cl}$ for difference } & \multirow[t]{2}{*}{$p$-value } \\
\hline & & & & Lower & Upper & \\
\hline \multirow{3}{*}{ Music } & \multirow{3}{*}{ PR } & Pre day $1 \&$ post day 1 & 10.68 & 2.16 & 19.21 & 0.01 \\
\hline & & Pre day 1 \&post day 2 & 6.81 & -0.53 & 14.16 & 0.07 \\
\hline & & Post day $1 \&$ post day 2 & -3.87 & -11.51 & 3.76 & 0.58 \\
\hline \multirow{3}{*}{ Music } & \multirow{3}{*}{ RR } & Pre day $1 \&$ post day 1 & 0.750 & -1.55 & 3.05 & 1.00 \\
\hline & & Pre day 1 \&post day 2 & 4.06 & 0.210 & 7.92 & 0.04 \\
\hline & & Post day $1 \&$ post day 2 & 3.31 & -1.23 & 7.86 & 0.21 \\
\hline \multirow{3}{*}{ Music with Yoga } & \multirow{3}{*}{ RR } & Pre day $1 \&$ post day 1 & $1.85^{*}$ & 0.040 & 3.67 & 0.04 \\
\hline & & Pre day 1 \&post day 2 & $3.92^{*}$ & 1.41 & 6.45 & 0.001 \\
\hline & & Post day $1 \&$ post day 2 & 2.07 & -0.29 & 4.44 & 0.09 \\
\hline
\end{tabular}

with yoga group, SBP, DBP \& PR were not significantly changed in two days but RR \& anxiety were changed from 24 to 20 and 0.94 to 0.29 respectively. $(\mathrm{p}=0.001$, $\mathrm{p}=0.001)$.(Table 2).

A post-hoc test for PR and RR for music group and $\mathrm{RR}$ for music with yoga group was administered to find out the possible combinations of effect occurred during these two days. Pulse rate was significantly reduced on day1 post assessment and it was significant $(\mathrm{p}=0.01)$. RR was significantly reduced on second day post assessment compared to day 1 pre assessment $(\mathrm{p}=0.04)$. In music with yoga group, $\mathrm{R} R$ was reduced significantly from day 1 pre compared to day 1 post $(\mathrm{p}=0.04)$ and also day 1 pre compared with day 2 post $(\mathrm{p}=0.001)$. (Table 3).

\section{DISCUSSION}

Music listening has been consistently reported to bring down anxiety in patients through stress reducing effects $^{5,14-16}$ and more specifically in cardiac patients as per the latest Cochraine review of patients with coronary heart disease. ${ }^{17}$ The results of our study is in consonance with these findings. Another mechanism put-forth for music listening to bring down anxiety is that music can help people focus their attention away from distressing situations and generate positive moods and emotions which in turn reduces the anxiety levels. ${ }^{17}$ Similarly, the music listening interventions also stabilized the physiological responses by reducing the SBP, DBP, $\mathrm{PR}$ and RR . Both the reduction of anxiety and the regularization of the physiological responses could also be attributed to the music listening intervention suppressing the sympathetic activity leading to reduced adrenergic activity and regulation of the autonomic cardiovascular rhythms. ${ }^{18,19}$

For this study, the researcher prepared a play list of Indian classical music in the form of raga improvisation from which the patient chose one musical piece for the music listening intervention. All the improvisational pieces had the common characteristics of slow tempo, soft timbre (flute), simple melodic phrases in the same octave without sudden jumps of octaves and with low emotional intensity. All the patients participated in the study also were belonging to the same ethnic group.

In our study, the combination of music and the pranava pranayama intervention also consistently reduced the anxiety of patients posted for angiography during the period of intervention. The mechanism for reduction of stress and anxiety by pranava pranayama is also the same as that for music. The pranava pranayama intervention also improved the deep breathing pattern of the patients facilitating relaxation. The findings of our study indicated that music therapy as a standalone adjuvant therapy was able to reduce the anxiety of the patients and also stabilized the physiological responses. Also, the combination of music and yoga therapies could produce the desired result of reducing the anxiety and enhancing deep breathing thereby facilitating relaxation.

An important limitation of the study was that though the sample size calculated was 30 in each group, 
the required number of samples were not registered in the cardio-vascular ward for angiography during the entire study period of 4 months and in total only 45 patients could be recruited for the study.

\section{CONCLUSION}

Music therapy interventions are beneficial to reduce anxiety and physiological disturbances in patients undergoing coronary angiography. Combination of music and yoga therapies can reduce anxiety and regularize breathing patterns in these patients. However, more studies are needed to confirm these findings with the combination of these music and yoga treatment strategies.

\section{CONFLICTS OF INTEREST}

None

\section{ACKNOWLEDGEMENTS}

We thank Mrs. Bhuvaneswari Ramesh, Tutor, CMTER for her valuable assistance during the study period

\section{References}

1. Woodyard C. Exploring the therapeutic effects of yoga and its ability to increase quality of life. Int J Yoga. 2011;4:49-54.

2. Scott A. Managing anxiety in ICU patients: the role of pre-operative information provision. NursCrit Care. 2004;9:72-9.

3. Delewi R, Vlastra W, Rohling WJ, Wagenaar TC, Zwemstra M, Meesterman MG et al. Anxiety levels of patients undergoing coronary procedures in the catheterization laboratory. Int J Cardiol. 2017;228:926-30.

4. Pritchard MJ. Identifying and assessing anxiety in pre-operative patients. Nurs Stand. 2009;23:35-40.
5. Keegan L. Therapies to reduce stress and anxiety. Crit Care Nurs Clin North Am. 2003;15:321-7

6. Bradt J, Dileo C, Shim M. Music interventions for preoperative anxiety. Cochrane Database Syst Rev. 2013;(6) :CD006908

7. Bhavanani AB, Madanmohan, Sanjay Z, Vithiyalakshmi L. Immediate cardiovascular effects of pranava relaxation in patients of hypertension and diabetes. Biomed Hum Kinet.2012; 4:66-9.

8. Bhavanani AB, Madanmohan, Sanjay Z, Basavaraddi IV. Immediate cardiovascular effects of pranava pranayama in hypertensive patients. Indian J Physiol Pharmacol. 2012;56:273-8.

9. Sharma VK, Trakroo M, Subramaniam V, Rajajeyakumar M, Bhavanani AB, Sahai A. Effect of fast and slow pranayama on perceived stress and cardiovascular parameters in young healthcare students. Int J Yoga 2013;6:104-10

10. Vempati RP, Telles S. Yoga based guided relaxation reduces sympathetic activity in subjects based on baseline levels. Psychol Rep. 2002; 90:487-94.

11. Aldridge $\mathrm{K}$. The use of music to relieve pre-operational anxiety in children attending day surgery. Australian J Music Ther.1993;4:17.

12. Palmer JB, Lane D, Mayo D, Schluchter M, Leeming R. Effects of music therapy on anesthesia requirements and anxiety in women undergoing ambulatory breast surgery for cancer diagnosis and treatment: A randomized controlled trial. J Clin Oncol. 2015;33:3162-8.

13. Davey HM, Barratt AL, Butow PN, Deeks JJ. A one-item question with a Likert or visual analog scale adequately measured current anxiety. J Clin Epidemiol. 2007;60:356-60.

14. Khemka SS., Rao NH, Nagarathna R. Immediate effects of two relaxation techniques on healthy volunteers. Indian J.Physiol. Pharmacol. 2009; 53:67-72.

15. Thoma MV, La Marca R, Brönnimann R, Finkel L, Ehlert U, Nater UM. The effect of music on the human stress response. PLoS One. 2013; (8):e70156.

16. Ventura T, Gomes MC, Carreira T. Cortisol and anxiety response to a relaxing intervention on pregnant women awaiting amniocentesis. Psychoneuroendocrinology. $2012 ; 37: 148-56$

17. Bradt J, Dileo C, Potvin N. Music for stress and anxiety reduction in coronary heart disease patients. Cochrane Database Syst Rev. 2013 Dec 28;(12):CD006577

18. Nilsson $U$. The effect of music intervention in stress response to cardiac surgery in a randomized clinical trial. Heart Lung. 2009;38:201-7.

19. Gillen E, Biley F, Allen D. Effects of music listening on adult patients' pre-procedural state anxiety in hospital. Int J Evid Based Healthc. 2008;6:24-49. 